\title{
Full Wave Characterization of a Through Hole Via
}

Show-Gwo Hsu and Ruey-Beei Wu

Rm. 509, Department of Electrical Engineering National Taiwan University, Taipei, Taiwan, R.O.C. FAX : 886-2-3671909, TEL : 886-2-3635251 Ext 276

\begin{abstract}
The frequency dependent propagation characteristics of a via penetrating through a hole in the ground plane were investigated by a numerical approach which combines moment method and matrix pencil method to extract the scattering parameters.
\end{abstract}

$0-7803-1427-1 / 93 / \$ 3.00$ (C) 1993 IEEE 


\title{
Full Wave Characterization of a Through Hole Via
}

\author{
Show-Gwo Hsu and Ruey-Beei Wu \\ Rm. 509, Department of Electrical Engineering \\ National Taiwan University, Taipei, Taiwan, R.O.C \\ FAX : 886-2-3671909, TEL : 886-2-3635251 Ext 276
}

\section{Introduction}

The miniaturization and large scale integration of electronic devices places increasing demand on the multi-layer interconnection geometry. In multi-layer structures, there are parallel lines for the signal transmission as well as the vias to connect the signal lines in different layers. Physically, the discontinuity due to the via will introduce the electromagnetic energy storage and radiation. At low frequencies, the via size is much smaller than the wavelength and the radiation is negligible [1]. As the clock rates in electronic circuits and the operating frequencies in communication systems become ever increasing, the electric and magnetic energy near the discontinuities will strongly couple to each other and result in significant amount of electromagnetic radiation. Some studies based on the finite-difference time-domain method [2] [3] and the full wave analysis in the frequency domain [4] were presented in the literature.

This paper deals with the propagation characteristics of a via through a hole in the ground plane. Numerical results are presented to investigate the frequency dependent propagation characteristics for the via structures with various wire diameters and via heights.

\section{Statement of the Problem}

Fig. 1 shows a typical through hole via structure. The incident waves are TEM modes of amplitudes $A_{1}$ and $A_{2}$ propagating in the upper and lower transmission lines, respectively. It is of interest to calculate the amplitudes $B_{1}$ and $B_{2}$ of the reflected TEM waves in the transmission lines due to the presence of the via. The problem can be decomposed into two parts. One is an antenna problem and the other is a short circuit problem. For either problem, only the original structure in a half space requires consideration. Let the semi-infinitely long transmission line be truncated to a certain length $L$. By the image theory, the ground plane can be replaced by including the image via and transmission line in the other half space and imposing a frill magnetic current of $2 \vec{M}$ on the via hole. Under the thin wire approximation, the current distribution on the via and a section of transmission line is solved by the moment method and the scattering parameters are extracted by the matrix pencil method [5].

\section{Numerical Examples}

For simplicity, only the numerical examples of symmetrical structures with via height $h_{1}=h_{2}=h$ and transmission line radius $a_{1}=a_{2}$ are presented here, although the analysis can handle the asymmetrical structures without any difficulty. Assume that a TEM mode of unit amplitude is incident from the line $1\left(A_{1}=1\right)$ while line 2 is terminated with a matched load $\left(A_{2}=0\right)$.

Fig. 2 shows the the phase and magnitude of the reflection coefficient $\Gamma$ for the via structures with $a_{1} / a=0.75, b / a=2$, and $h / a_{1}=20$ or 30 . The solid curves are obtained by the present analysis. It is found that the phase is about $-90^{\circ}$ at quasistatic limit and decreases almost linearly versus the frequency. This implies that the via structure is dominantly capacitive. Also, a resonance phenomenon where $\Gamma$ reaches its maximum happens at the frequency of $k h \simeq 0.65$, i.e., the via height $h$ is about one 
tenth of the wavelength.

The dashed curves also included in Fig.2 for comparison are based on the quasistatic model [1]. As compared with our full wave analysis, both results are in good agreement at low frequencies and present a similar resonance phenomenon except for a slight frequency shift. However, the quasi-static model deteriorates above the resonance frequency and fails to predict the behavior of $\Gamma$ properly. The reflectionless phenomenon predicted by the quasi-static analysis will not occur in light of the nonnegligible radiation resistance at higher frequencies. Quantitatively, the results show that the quasi-static model is valid until $k h=0.4$, which corresponds to an operating frequency of $6.4 \mathrm{GHz}$ if the via height is $h=3 \mathrm{~mm}$.

Fig. 3 shows the propagation characteristics versus the frequency for the via structures with $a_{1} / a=1, b / a=2$, and $h / a$ as a parameter. At the low frequency limit, it is found that $|\Gamma| \rightarrow 0,|T| \rightarrow 1$, and the radiation loss tends to zero, as expected intuitively. Although the reflection is smaller as the frequency goes higher than the resonance frequency, the transmission coefficient always decreases and radiation loss increases versus the frequency. The curve with $h / a=30$ shows that the radiation loss may become as high as 0.4 (or $4 \mathrm{db}$ ) at $k h=1$, which corresponds to an operating frequency of 16.7 $\mathrm{GHz}$ if the via height is $h=3 \mathrm{~mm}$.

Fig. 4 presents the propagation characteristics versus the via height $h$ while choosing via radius $a$ as the parameter. The operating frequency is taken to be $10 \mathrm{GHz}$ and the radius of the transmission line is $a_{1}=50 \mu \mathrm{m}$. The reflection and radiation loss can be reduced slightly by choosing a smaller via radius $a$.

\section{Conclusions}

In this study, a full wave approach is presented to deal with the propagation characteristics of a through hole via discontinuity. Numerical results are included to discuss the frequency dependence of the propagation characteristics and the effects due to various geometric parameters of the via structure. Roughly speaking, the radiation loss may be as high as 0.4 if the via height is larger than $0.16 \lambda$. To reduce the radiation loss and achieve better transmission characteristics, this study recommends choosing a shorter and thinner via.

\section{References}

[1] T. Y. Wang, R. F. Harrington, and J. R. Mautz, "Quasi-static analysis of a microstrip via through a hole in a ground plane," IEEE Trans. Microwave Theory Tech., vol. MTT-36, pp. 1008-1013, June 1988.

[2] S. Maeda, T. Kashiwa, and I. Fukai, "Full wave analysis of propagation characteristics of a through hole using the finite-difference time-domain method," IEEE Trans. Microwave Theory Tech., vol. MTT-39, pp. 2154-2159, Dec. 1991.

[3] W. D. Becker, P. H. Harms, and R. Mittra, "Time-domain electromagnetic analysis of interconnects in a computer chip package," IEEE Trans. Microwave Theory Tech., vol. MTT-40, pp. 2155-2163, Dec. 1992.

[4] R. Sorrentino, F. Alessandri, M. Mongiardo, G. Avitabile, and L. Roselli, "Fullwave modeling of via hole grounds in microstrip by three-dimensional mode matching technique" IEEE Trans. Microwave Theory Tech., vol. MTT-40, pp. 2228-2234, Dec. 1992.

[5] Y. B. Hua and T. K. Sarkar, "Generalized pencil-of-function method for extracting poles of an EM system from its transient response," IEEE Trans. Antennas Propagat., vol. AP-37, pp. 229-234, Feb. 1989. 


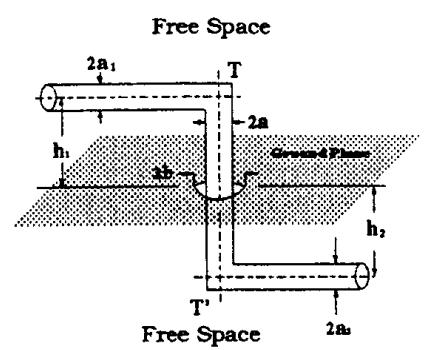

Figure 1: A typical through hole via structure.

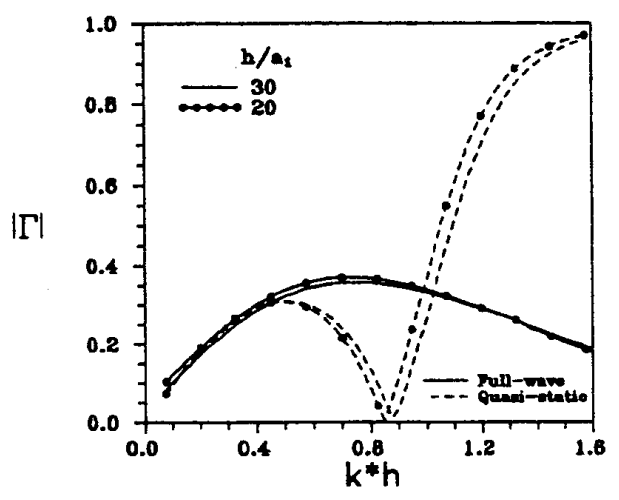

(a)

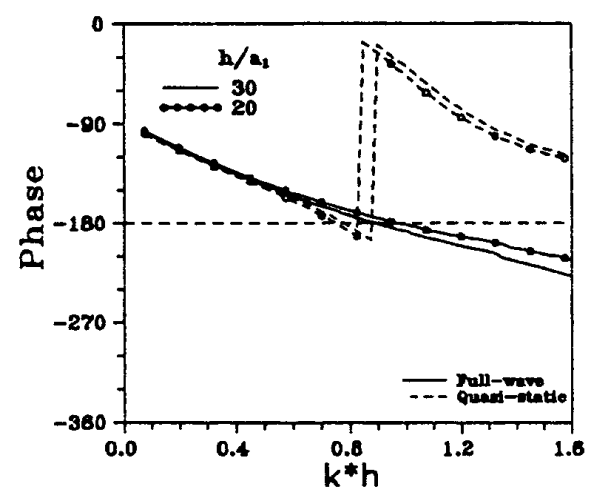

(b)

Figure 2: Comparsion between the ful1 wave anlaysis and the quasi-static model which are denoted by solid curves and dashed curves, respectively, (a) amplitude, (b) phase. The via structures have parameters $a_{1} / a=0.75, b / a=2.0$ and $h / a_{1}=20$ or 30 .

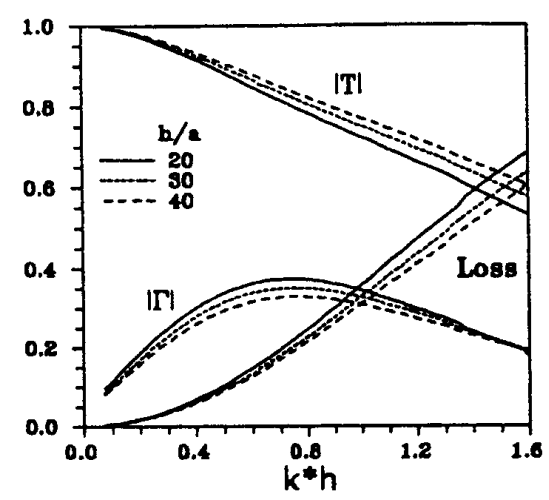

Figure 3: The propagation characteristics versus the frequency for the via structures with $a_{1} / a=1, b / a=2.0$ and $h / a$ as a parameter.

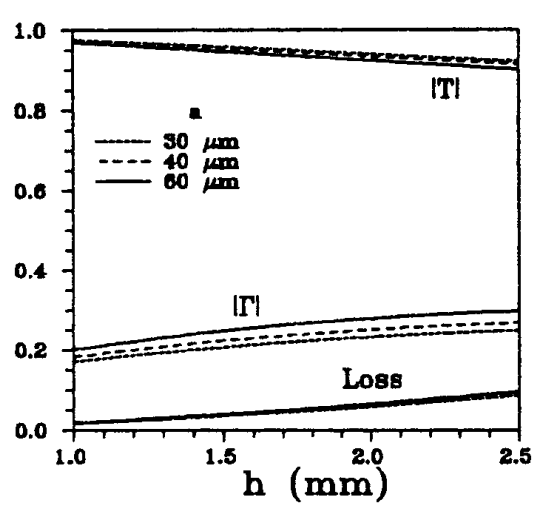

Figure 4: The propagation characteristics at $10 \mathrm{GHz}$ versus $h$ for the via structures with $a_{1}=50 \mu \mathrm{m}, b=100 \mu \mathrm{m}$, and $a=30,40$, or $60 \mu \mathrm{m}$. 